Research Article

\title{
Research on Bifurcation Response for Vortex-Induced Vibration of Top Tension Riser in Shear Flow
}

\author{
Yuancen Wang $\mathbb{D}^{1,2}$ Zhiqiang $W u\left(\mathbb{D},,^{1,2}\right.$ and Xiangyun Zhang $\mathbb{D}^{1,2,3}$ \\ ${ }^{1}$ School of Mechanical Engineering, Tianjin University, Tianjin 300350, China \\ ${ }^{2}$ Tianjin Key Laboratory of Nonlinear Dynamics and Chaos Control, Tianjin 300350, China \\ ${ }^{3}$ Tianjin Sino-German University of Applied Sciences, Tianjin 300350, China
}

Correspondence should be addressed to Zhiqiang Wu; zhiqwu@tju.edu.cn

Received 10 July 2019; Revised 8 August 2019; Accepted 14 August 2019; Published 4 November 2019

Academic Editor: Jian G. Zhou

Copyright (c) 2019 Yuancen Wang et al. This is an open access article distributed under the Creative Commons Attribution License, which permits unrestricted use, distribution, and reproduction in any medium, provided the original work is properly cited.

\begin{abstract}
The top tension riser (TTR) is one of the most frequently used equipment in deep-sea petroleum engineering. At present, the research methods of its vortex-induced vibration (VIV) are mainly focused on finite element analysis and experiment. The understanding of its various nonlinear mechanical mechanisms would be inadequate via limited numerical or experimental studies rather than nonlinear analysis of its rich dynamics. Based on the Van der Pol wake oscillator model, the nonlinear dynamic model of the TTR subject to shear flow VIV is established. The proposed model includes the fluid-structure interaction of the TTR under shear flow. Dynamic behavior of the TTR in association with the variation of flow velocity is investigated. The dynamic behavior is simulated by computing the local maximum displacement response via the fifth-order Galerkin discretization. The Poincare map is then utilized to quantify the dynamic property of TTR under each individual flow velocity, which helps identifying the bifurcation path of the nonlinear system. The time history, phase diagram, FFT spectrum, and envelope diagram about the riser VIV at typical flow velocity in different regions of the bifurcation diagram are then given. It is found that the VIV response of the TTR depicts the Hopf bifurcation phenomena with bistable characteristics. Together with the structural eigenanalysis and the three-dimensional spectrum contour, the main dynamic features of the TTR in shear flow are more comprehensively understood. Such understandings may provide new ideas and references for the design and optimization of the riser structural parameters.
\end{abstract}

\section{Introduction}

With the increasing demand for oil and gas resources globally, the exploitation of marine oil and gas resources gradually extend from shallow to deep water. As the deepsea risers are slender with large aspect ratio, the natural frequency and structure stability of the riser are reduced, and the vortex-induced vibration (VIV) of the deep-sea riser depicts more complicated dynamics with increasing water depth. Under this trend, a lot of new problems and new phenomena are emerged [1]. At present, the dynamic studies of the deep-sea riser are mainly concentrated on the field of VIV. VIV is essentially a nonlinear, self-excited, and selflimited multi-degree-of-freedom resonance response due to the nonlinearities existed either in structure or fluid side, for example, the lock-in response, hysteresis, displacement jump, bifurcation, and chaos [2]. Vortex shedding occurs when the sea current flows through the riser. When the vortex shedding frequency is close to the natural frequency of the riser, vortex-induced resonance will occur and may cause serious damage to the riser. The natural frequency of the riser directly affects the excitation of vortex-induced vibration and parametric vibration. Therefore, study of the riser eigenvalues that vary with respect to structural parameters is helpful to determine the dynamic characters and VIV responses of the riser.

In order to study the VIV phenomenon of slender structures subject to nonuniform flow field such as shear flow, researchers have conducted a series of large-scale riser experiments [3,4] and CFD simulations [5-7] since the 
1990s [8]. With the improvement of test conditions, largescale deep-water experiments have been greatly carried out in recent years. Huarte observed multimode VIV experimentally and measured the drag force coefficient [9]. Huse derived the axial displacement function of the riser by modal analysis [4]. After Lie and Kaasen analyzed the test data of Huse by the modal method, the modal weighting coefficient was obtained, and the weights of each mode contributing to vibration response are analyzed, which provide valuable experimental results for the study of multimode VIV [10]. Lucor et al. calculated that in the linear shear flow, the VIV response of the flexible cylinder has locked regions in the high flow velocity region [11]. Tang et al., Ghayesh et al., Modarres-Sadeghi, and Paidoussis et al. also studied the dynamic phenomena of fluid-conveying pipes subjected to internal axial flow [12-14]. This provides a lot of reference and help for the nonlinear dynamic research of riser VIV under working conditions in recent years [15-18].

From the analytical perspective, the most commonly used empirical model for analyzing VIV is the wake oscillator model. The wake oscillator model does not consider the details of the flow field and structure. It treats the oscillating structure and fluid as a whole system, and considers the wake as a nonlinear oscillator. The vibration of the wake oscillator induces the vibration of the structure; in turn, the vibration of the structure feeds back to affect the wake [2]. Based on the research of the nonlinear oscillator model, Hartlen and Currie found out that the Van der Pol equation can be used as the govern equation of the lift coefficient of the wake. Fluid-structure interaction can be solved jointly with the vibration equation of the structure included [19]. Mathelin and co-workers summarized the previous work and extended the Van der Pol wake oscillator model [20-23]. Based on the Van der Pol wake oscillator model, Guo and Lou had established the VIV model of TTR considering internal flow; the validity of the model was verified by model tests [24]. Tang studied the dynamic response for coupled parametric vibration and VIV of TTR combined with the Van der Pol wake oscillator model [25].

The present experimental and finite element analysis methods can measure and calculate the response characteristics of risers at specific flow velocity. And Wanderley and Navrose have found some bistable behaviors and bifurcation phenomenon of cylinder in experiments and CFD simulations [26, 27]. However, it is difficult to investigate the continuous change of VIV steady-state response of the riser in larger flow velocity ranges. Moreover, the characteristics of VIV response at specific flow velocity cannot be directly judged from the traditional response diagram of flow velocity and riser amplitude. The observation of such trend is significant on the study of the multistable phenomenon and natural vibration characteristics in shear flow. This paper takes the top tension riser (TTR) of the spar platform as the research object. The Van der Pol wake oscillator equation is used to describe the fluid force of the TTR fluid-structure coupled system. Based on the bending vibration theory of the Euler-Bernoulli beam and the high-order Galerkin discretization scheme, the influence of the sea flow velocity on the nonlinear dynamic behavior of the riser system is analyzed by Poincaré map method.

\section{Dynamical Model}

For flexible TTR with large aspect ratio, we usually assume that the riser is vertically supported by the platform, and the body has a uniform circular cross section along the axial direction. The lower end of the riser is hinged on the universal joint, while the top end of the riser has top tension applied by the tensioner. The mechanical layout can be regarded as a simply supported beam with top tension for theoretical model development. Assuming the VIV is caused by the linear shear flow, the physical model of the TTR is shown in Figure 1.

When the riser VIV appears, its amplitude in the crossflow direction ( $y$ direction) is generally much larger than the amplitude in the in-line direction ( $x$ direction), especially when the riser VIV "lock-in" phenomenon occurs. In this paper, we mainly study the cross-flow vibration problem of the riser VIV. Based on the bending vibration theory of the Euler-Bernoulli beam [28], the motion equation of TTR along the cross-flow direction ( $y$ direction) could be expressed as

$$
\begin{aligned}
\mathrm{EI} & \cdot \frac{\partial^{4} y(z, t)}{\partial z^{4}}-\frac{\partial}{\partial z}\left(T(z) \cdot \frac{\partial y(z, t)}{\partial z}\right) \\
& +\left(m_{r}+m_{f}+m_{a}\right) \frac{\partial^{2} y(z, t)}{\partial t^{2}}+C \cdot \frac{\partial y(z, t)}{\partial t}=F_{y}
\end{aligned}
$$

where $y(z, t)$ is the cross-flow displacement of the riser and is a continuous function of the vertical displacement $z$ and the time $t$ about the coordinate axis. EI is the bending stiffness; $T$ is the top tension force, which can be expressed as $T(z)=A_{s} g\left(\rho_{s}-\rho_{w}\right)\left(f_{\text {top }} L-z\right)$, where $A_{s}$ is the riser crosssectional area $\left(A_{s}=\pi^{2}\left(D^{2}-d^{2}\right) / 4 ; D\right.$ is the outer diameter and $d$ is the inner diameter of the riser); $\rho_{s}$ and $\rho_{w}$ are the riser material density and seawater density, respectively; $f_{\text {top }}$ is the top tension coefficient, and $L$ is the length of the riser; $m_{r}$ is the riser quality per unit length $\left(m_{r}=\pi \rho_{s}\left(D^{2}-d^{2}\right) / 4\right)$; and $m_{f}$ is the riser fluid quality per unit length $\left(m_{f}=\right.$ $\left.\rho_{f} \pi d^{2} / 4\right), m_{a}$ is the additional quality per unit length $\left(m_{a}=\right.$ $C_{a} \rho_{w} \pi D^{2} / 4$ in which $C_{a}$ is the added mass coefficient), and $C$ is structural damping $[25,29]$.

In this paper, the Van der Pol equation is used to represent the vibration characteristics of the wake vortex shedding. At the same time, inertial coupling is used to express the interaction between the riser structure and the fluid. The equation is as follows [22]:

$$
\ddot{q}+\varepsilon \Omega_{f}\left(q^{2}-1\right) \dot{q}+\Omega_{f}^{2} q=\frac{A}{D} \ddot{y} .
$$

The variable $q$ represents the ratio of the instantaneous lift coefficient $C_{L}$ of the local fluid to the structure to the static lift coefficient $C_{L 0}$ of the structure, namely, $q=2 C_{L} / C_{L 0} . \Omega_{f}$ is the vortex shedding frequency, $\Omega_{f}=2 \pi \mathrm{St} U / D$, and $S t$ is the Strouhal number. $A$ and $\varepsilon$ are the coupling coefficient determined by the test, generally taking $A=12, \varepsilon=0.3$ [30].

The fluid force which applies to the pipeline includes two parts, the vortex lift and the damping force.

$$
F_{y}=f_{y}+f_{y}^{\prime} .
$$




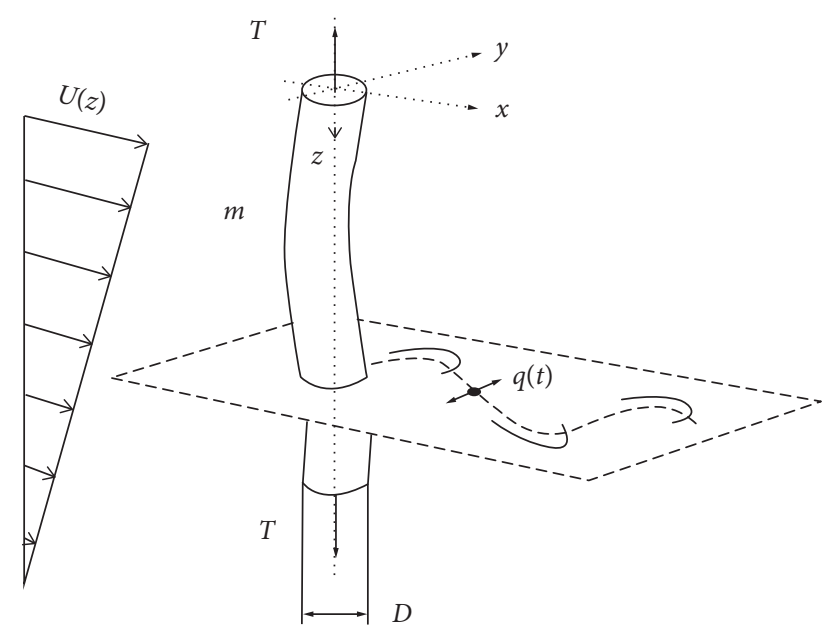

Figure 1: Schematic diagram of the riser VIV.

The vortex lift is

$$
f_{y}=\frac{1}{2} \rho_{w} U_{f}^{2} D \frac{C_{L 0}}{2} q .
$$
as

The damping force is expressed by the Morison equation

$$
f_{y}^{\prime}=\frac{1}{2} \rho_{w} D C_{D} \dot{y} U_{f}
$$

where $C_{D}$ is the drag coefficient and $U_{f}$ is the sea current velocity as $U_{f}=(1-(z / L)) U$, in which $U$ is the maximum velocity occurring at the sea surface. It is assumed that the minimum velocity at the seafloor is 0 , and the velocity decreases linearly from the sea surface to the seafloor.

Based on the various equations given above, the VIV cross-flow govern equations of the TTR subject to shear flow are obtained as

$$
\left\{\begin{array}{l}
\mathrm{EI} \frac{\partial^{4} y(z, t)}{\partial z^{4}}-\frac{\partial}{\partial z}\left[T(z, t) \cdot \frac{\partial y(z, t)}{\partial z}\right] \\
\quad+\left(m_{r}+m_{f}+m_{a}\right) \frac{\partial^{2} y(z, t)}{\partial t^{2}}+C \frac{\partial y(z, t)}{\partial t} \\
\quad=\frac{1}{2} \rho_{w} U_{f}^{2} D \frac{C_{L 0}}{2} q-\frac{1}{2} \rho_{w} D C_{D} \dot{y} U_{f}, \\
\ddot{q}+\varepsilon \Omega_{f}\left(q^{2}-1\right) \dot{q}+\Omega_{f}^{2} q=\frac{A}{D} \ddot{y} .
\end{array}\right.
$$

Riser boundary conditions are as follows:

$$
\begin{array}{r}
y(0, t)=y(L, t)=0, \\
\left.\frac{d^{2} y}{d z^{2}}\right|_{z=0, z=L}=0 .
\end{array}
$$

Letting $\bar{y}=y / D, \quad \bar{z}=z / L, \quad M=m_{r}+m_{f}+m_{a}, \quad$ and substituting these transformations into (6), the dimensionless model is as follows:

$$
\left\{\begin{array}{c}
\alpha_{5} \frac{\partial^{4} \bar{y}}{\partial \bar{z}^{4}}+\alpha_{1} \frac{\partial \bar{y}}{\partial \bar{z}}-\alpha_{1}\left(f_{\text {top }}-\bar{z}\right) \frac{\partial^{2} \bar{y}}{\partial \bar{z}^{2}}+\frac{\partial^{2}(\bar{y})}{\partial t^{2}}+\alpha_{2} \frac{\partial \bar{y}}{\partial t} \\
=\alpha_{3}(1-\bar{z})^{2} U^{2} q-\alpha_{4} \frac{\partial \bar{y}}{\partial t}(1-\bar{z}) U \\
\frac{d^{2} q}{d t^{2}}+\varepsilon \Omega_{f} q^{2} \frac{d q}{d t}-\varepsilon \Omega_{f} \frac{d q}{d t}+\Omega_{f}^{2} q=A \frac{\partial^{2} \bar{y}}{\partial t^{2}}
\end{array}\right.
$$

where $\alpha_{1}=A_{s} g\left(\rho_{s}-\rho_{w}\right) / M L, \alpha_{2}=C / M, \alpha_{3}=\rho_{w} C_{L 0} / 4 M$, $\alpha_{4}=\rho_{w} D C_{D} / 2 M$, and $\alpha_{5}=\mathrm{EI} / M L^{4}$.

In this paper, the Galerkin method is used to discretize the partial differential equation. Assume the solution with the following form [31]:

$$
\begin{aligned}
& \bar{y}=\sum_{i=1}^{n} \varphi_{i}(\bar{z}) \bar{y}_{i}(t), \\
& q=\sum_{i=1}^{n} \varphi_{i}(\bar{z}) q_{i}(t),
\end{aligned}
$$

where $\bar{y}_{i}(t)$ and $q_{i}(t)$ are the generalized coordinates of the riser and wake oscillator displacements, respectively. $\varphi_{i}(\bar{z})$ is the $i$ th order mode function with $\varphi_{i}(\bar{z})=\sqrt{2} \sin (i \pi \bar{z})$. Substituting them into equation (8) and multiplying the mode function $\varphi_{j}(\bar{z})$ at both ends of the equations, the equations from 0 to 1 are integrated. The $n$th order VIV differential equations of the riser system can be obtained as follows:

$$
\left\{\begin{array}{l}
\ddot{\bar{y}}_{i}+\sum_{j=1}^{n} \bar{y}_{j} \int_{0}^{1}\left[\alpha_{5} \phi_{j}^{(4)}+\alpha_{1}\left(\bar{z}-f_{\text {top }}\right) \phi_{j}^{\prime \prime}+\alpha_{1} \phi_{j}^{\prime}\right] \phi_{i} d \bar{z} \\
\quad+\alpha_{2} \sum_{j=1}^{n} \dot{\bar{y}}_{i} \int_{0}^{1} \phi_{i} \phi_{j} d \bar{z}=\alpha_{3} U^{2} \sum_{j=1}^{n} q_{j} \int_{0}^{1}(1-\bar{z})^{2} \phi_{i} \phi_{j} d \bar{z} \\
\quad-\alpha_{4} U \sum_{j=1}^{n} \dot{\bar{y}}_{i} \int_{0}^{1}(1-\bar{z}) \phi_{i} \phi_{j} d \bar{z} \\
\ddot{q}_{i}+\varepsilon \Omega_{f}\left(\sum_{j}^{n} \sum_{k}^{n} \sum_{l}^{n} q_{j} q_{k} \dot{q}_{l} \int_{0}^{1} \phi_{j} \phi_{k} \phi_{l} \phi_{i} d \bar{z}-\dot{q}_{i}\right) \\
+\Omega_{f}^{2} q_{i}=\frac{A L}{D} \ddot{\bar{y}}_{i}, \quad(i=1,2, \ldots, n) .
\end{array}\right.
$$

\section{Bistable Phenomenon of Riser VIV in Shear Flow}

In this paper, the VIV of the $1000 \mathrm{~m}$ long steel top tension riser is taken as an example. The main parameters are shown in the following Table $1[7,32]$ :

For the VIV govern equations of the riser system, the higher the number of modes taken for discretization, the more accurate the calculated response would be. On the other hand, the computation is more costly. The main purpose of this paper is to research the bifurcation and bistable phenomena in the cross-flow response of the riser VIV in shear flow and the nonlinear vibration response 
TABLE 1: Parameters of TTR.

\begin{tabular}{lcc}
\hline Parameter & Symbol & Values \\
\hline Elastic modulus & $E$ & $210 \mathrm{GPa}$ \\
Seawater density & $\rho_{w}$ & $1025 \mathrm{~kg} / \mathrm{m}^{3}$ \\
Material density & $\rho_{s}$ & $7850 \mathrm{~kg} / \mathrm{m}^{3}$ \\
Internal fluid density & $\rho_{f}$ & $800 \mathrm{~kg} / \mathrm{m}^{3}$ \\
Outer diameter & $D$ & $0.325 \mathrm{~m}$ \\
Inner diameter & $d$ & $0.305 \mathrm{~m}$ \\
Riser length & $L$ & $1000 \mathrm{~m}$ \\
Top tension coefficient & $f_{\text {top }}$ & 1.3 \\
Additional mass coefficient & $C_{a}$ & 1.0 \\
Strouhal number & $\mathrm{St}$ & 0.2 \\
Drag coefficient & $C_{D}$ & 1.2 \\
\hline
\end{tabular}

characteristics of the riser at different flow velocities. Under the condition that the vibration response accuracy is satisfied within the range of the calculated flow velocities, the fifthorder Galerkin discretization scheme is selected to calculate the VIV response of the riser system in this section.

3.1. Bifurcation and Bistable Phenomena of the Riser. Using the Poincaré map method and continuation method $[33,34]$, two bifurcation diagrams of the local maximum value of the VIV cross-flow dimensionless displacement at the midpoint of the TTR (500 $\mathrm{m}$ from the sea level) with respect to the flow velocity increasing and decreasing are shown in Figure 2. The local maximum value of vibration displacement can be used to quantify the vibration characteristics of the riser system when VIV reaches steady state at various flow velocities. When the vibration is periodic, the local maximum value of the vibration displacement is a single point; when the vibration is almost periodic or other motion, the local maximum value of the vibration displacement would be a series of points at the corresponding flow velocity. It can be seen from Figure 2 that when the shear flow velocity gradually increases (red circle line) and gradually decreases (black dot line), there are various vibration patterns in the bifurcation diagram.

From the overall view of Figure 2, when the flow velocity is less than $0.2 \mathrm{~m} / \mathrm{s}$, the system response between two adjacent periodic solutions forms a torus of almost periodic solutions. There is an obvious jump and hysteresis phenomenon in the transition process between the periodic solution and the almost periodic solution. When the flow velocity is in the range of $0.2 \mathrm{~m} / \mathrm{s}$ to $0.22 \mathrm{~m} / \mathrm{s}$, the vibration response of the riser system may appear large jump phenomenon with the amplitude suddenly increasing or decreasing. In these regions, the vibration responses of the riser system appear torus Hopf bifurcation and subcritical Hopf bifurcation, respectively, and there is bistable behavior.

At the same time, the bifurcation diagrams of the riser at different positions ( $250 \mathrm{~m}$ and $750 \mathrm{~m}$ below sea level) are plotted in Figures 3(a) and 3(b), respectively.

Comparing Figures 2 and 3, it can be observed that the vibration responses of the riser at different positions of the riser body are not exactly the same in shear flow. At each position of the riser body, the bistable region where VIV

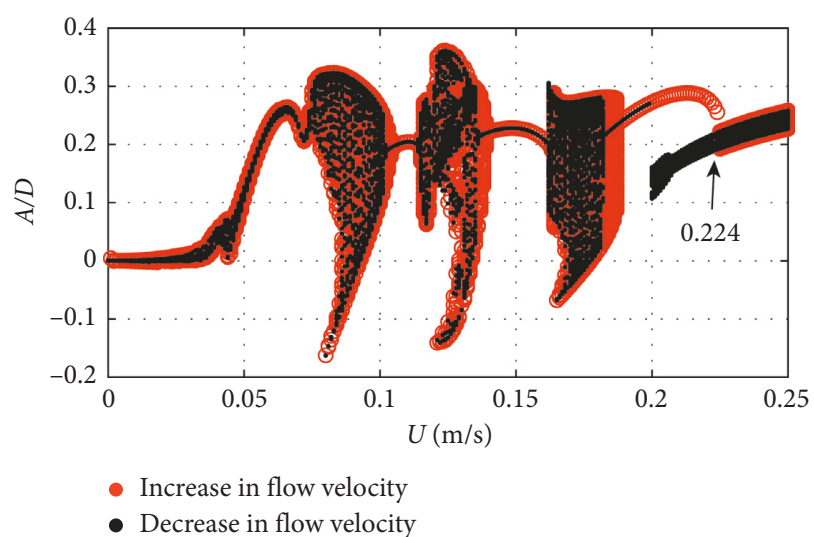

FIgURE 2: The bifurcation diagram of VIV displacement local maximum value at the midpoint.

present periodic and almost periodic vibration is basically the same, but the response characteristics are different.

3.2. The Modal Analysis of the Riser System. In this paper, the top tension riser is regarded as a nonlinear fluid-structure coupled system that interacts with the vortex induced by wake. The natural frequency of the riser coupled system is represented by the structural hydroelastic modal frequency. And the vortex shedding frequency caused by the wake is represented by the wake-induced modal frequency.

In order to analyze the VIV response conveniently and explore the changing law of riser modal frequency, this section calculates the eigenvalues of the Jacobian matrix at the equilibrium point $\left(y_{i}, \dot{y}_{i}, q_{i}, \dot{q}_{i}\right)=(0,0,0,0)$ of the riser system [35]. The varying trends of the structural hydroelastic modal frequency and the wake-induced modal frequency of the riser coupled system with respect to the flow velocity are obtained.

Figure 4 shows the change of the imaginary part of the eigenvalue of the riser system with the flow velocity in shear flow. For the riser fluid-structure coupled system, the imaginary part of eigenvalue can clearly show the trend of the structural hydroelastic modal frequency and the wakeinduced modal frequency. In Figure 4, the relatively flat curves are the structural hydroelastic modal frequency of the first five orders of riser structure; the curves with inclinations are the wake-induced modal frequencies for the first five orders.

3.3. Equivalent Vortex Shedding Frequency. The external excitation for the riser mainly comes from the vortex force caused by the sea current, and the strength and frequency of the vortex force are directly related to the flow velocity. Since the force of the shear flow acting on the riser is nonuniform, the strength and frequency of the excitation are not same along the riser. In order to predict the vortex-induced resonance, it is necessary to find the equivalent flow velocity and acting force of the shear flow to the riser.

Figure 5 is a schematic diagram of the equivalent flow velocity at the middle point of the riser in shear flow. 


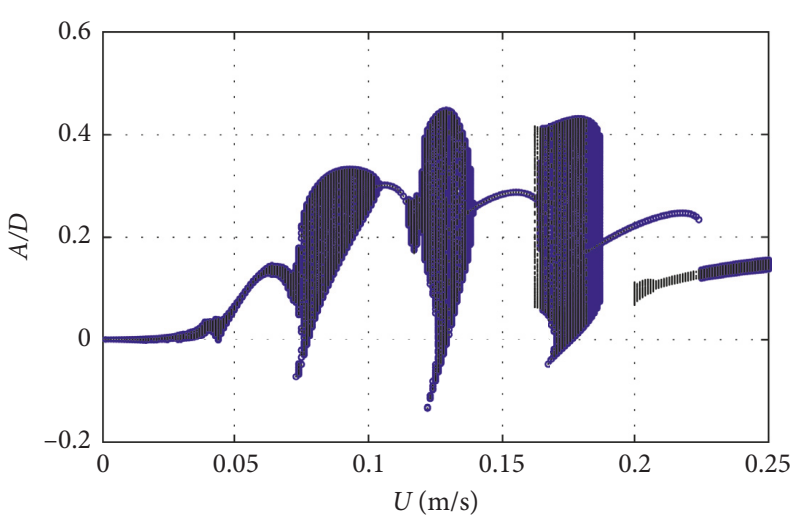

- Increase in flow velocity

- Decrease in flow velocity

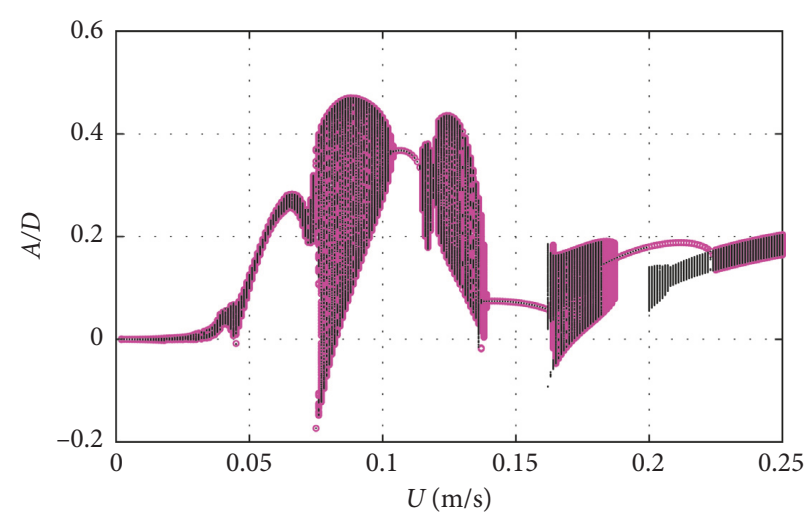

- Increase in flow velocity

- Decrease in flow velocity

Figure 3: The bifurcation diagram of VIV displacement local maximum value at different positions. (a) The position 250 meters from the top. (b) The position 750 meters from the top.

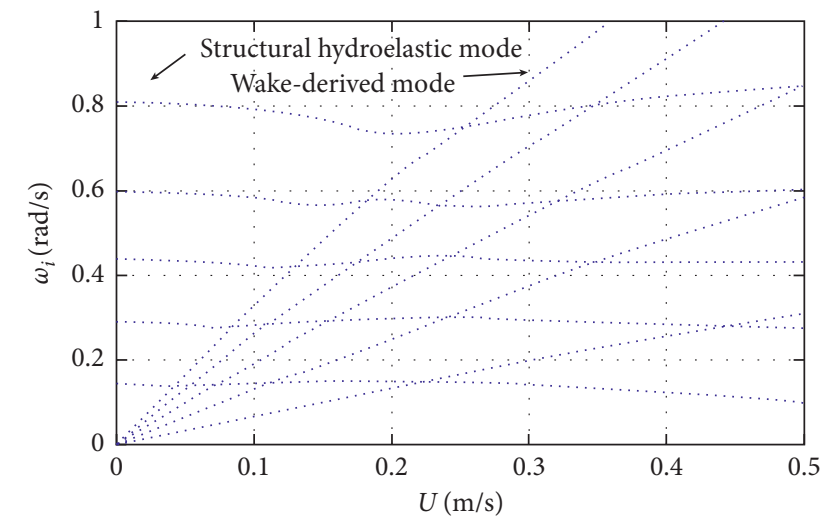

FIGURE 4: Imaginary part of eigenvalue of the riser system in shear flow.

Supposing $U$ is the maximum flow velocity of the sea surface, $U e$ is the equivalent flow velocity acting at the midpoint of the riser, and the seafloor flow velocity is zero. Because the larger the flow velocity, the greater the acting force, and the force can be treated proportional to flow velocity. For linear shear flow, assume that the trapezoid ABCD and the triangle $\mathrm{CDE}$ have equal area. The lengths of the line segments $\mathrm{AB}$ and CD represent the sizes of $U$ and $U e$, respectively, and the sum of $L 1$ and $L 2$ is the length $L$ of the riser. $U e=(\sqrt{2} / 2) U$ can be obtained by calculating the equivalent force of triangular distributed load. And the equivalent vortex shedding frequency of the linear shear flow is

$$
\Omega_{f e}=\frac{2 \pi S_{t} U e}{D}=\frac{\sqrt{2} \pi S_{t} U}{D} \text {. }
$$

\section{VIV Response Characteristics and Spectrum Analysis of the Riser System}

4.1. VIV Response Characteristics of the Riser System at Typical Flow Velocities. The response of VIV at the midpoint of the riser is calculated by choosing some typical flow velocities

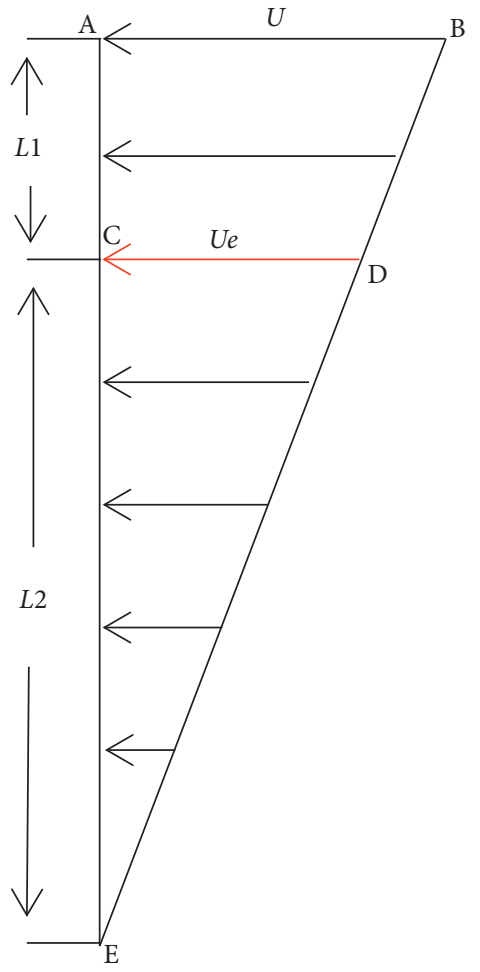

FIgURE 5: Schematic diagram of the equivalent flow velocity at the middle point of the riser in shear flow.

from different shape regions shown in Figure 2. Combined with displacement time history, phase diagram, Poincaré section, FFT spectrum, and maximum displacement envelope diagram, the response characteristics are illustrated. The blue dotted lines overlaid in the FFT spectrum shown in each figure (c) are the structural hydroelastic modal frequencies of the first five orders corresponding to the flow velocity as per Figure 4 . The vertical line marked with black stars in each figure (c) is the equivalent vortex shedding frequency at the midpoint of the riser. 


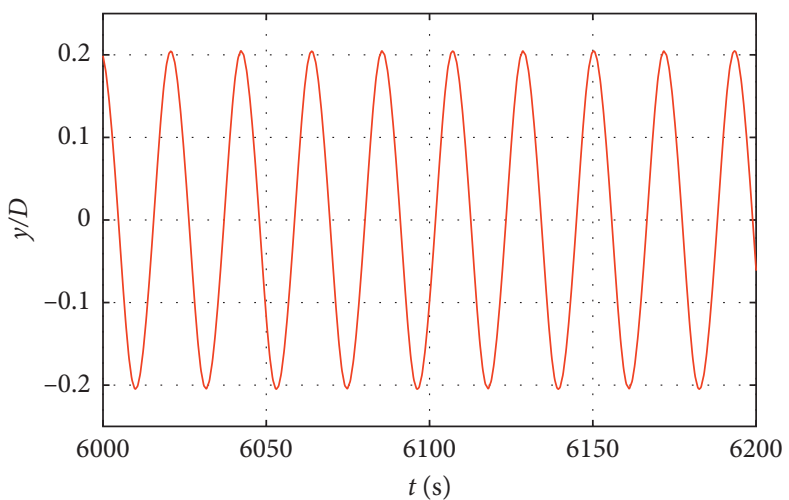

(a)

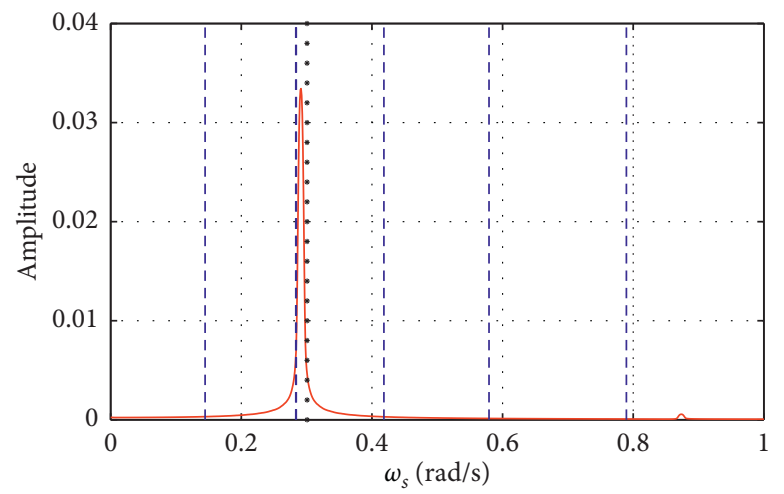

(c)

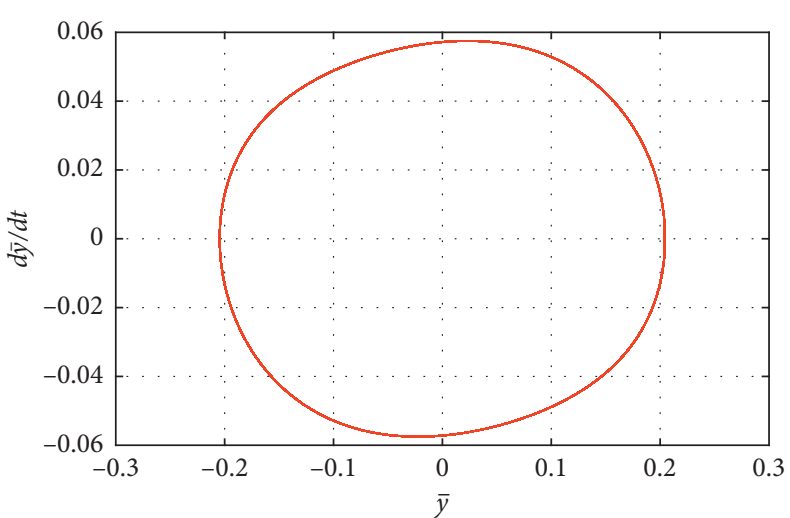

(b)

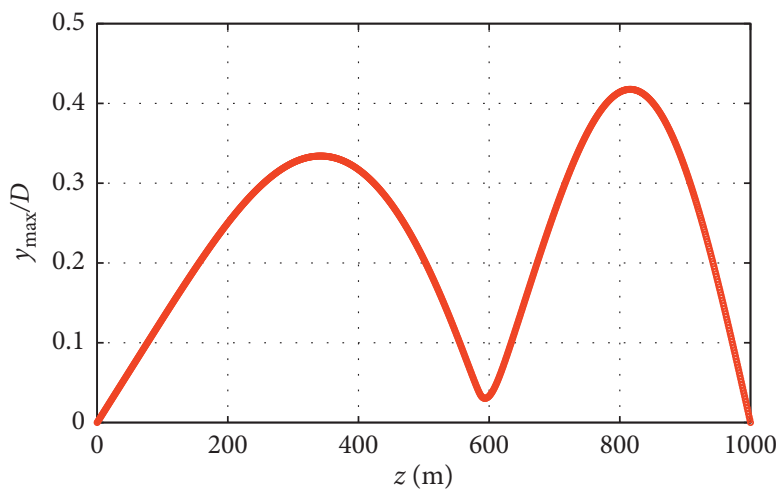

(d)

Figure 6: The VIV response of the riser system at surface flow velocity $U=0.11 \mathrm{~m} / \mathrm{s}$. (a) Displacement time history. (b) Phase diagram of displacement and velocity. (c) FFT spectrum. (d) Maximum displacement envelope.

In addition, when the velocity corresponding to the firstorder mode after Galerkin discretization exists $\dot{\bar{y}}_{1}=0$, the Poincare section is intercepted and projected into the phase diagram of displacement and velocity shown in figure (b) of Sections 4.1.2 and 4.1.3. If the Poincaré section has only one fixed point or a few discrete points, the system motion is periodic. When the Poincare section is a closed curve, the system motion is almost periodic, and when the Poincaré section has a group of irregular and dense points, the system motion may be chaotic.

4.1.1. Periodic Vibration. After analyzing Figure 2, it can be found out that under several flow velocity ranges from $0.106 \mathrm{~m} / \mathrm{s}$ to $0.113 \mathrm{~m} / \mathrm{s}, 0.141 \mathrm{~m} / \mathrm{s}$ to $0.16 \mathrm{~m} / \mathrm{s}$, and $0.188 \mathrm{~m} / \mathrm{s}$ to $0.199 \mathrm{~m} / \mathrm{s}$, there is only one local maximum for both forward and inverse sweeping of flow velocity. Also, such maxima are the same for both sweeping directions. This implies that the VIV response of the riser system at these flow velocities is periodic. Within these velocity ranges, two typical velocities are selected to calculate the VIV response of the riser as follows:

(1) $U=0.11 \mathrm{~m} / \mathrm{s}$ and $\Omega_{f e}=0.3 \mathrm{rad} / \mathrm{s}$

When $U=0.11 \mathrm{~m} / \mathrm{s}, \Omega_{f e}$ is near the primary resonance frequency of the $2^{\text {nd }}$-order structural hydroelastic mode. It can be found out that the phase diagram is elliptical. This shape indicates that the system response is periodic, which can be further confirmed from Figures 6(a) and 6(b). Note from Figures 6(a) and 6(b) that there is only one frequency component in the spectrum which is very close to the $2^{\text {nd }}$-order structural hydroelastic modal frequency. The maximum displacement envelope diagram is also shown as the $2^{\text {nd }}$-order resonance mode shape.

(2) $U=0.15 \mathrm{~m} / \mathrm{s}$ and $\Omega_{f e}=0.41 \mathrm{rad} / \mathrm{s}$

When $U=0.15 \mathrm{~m} / \mathrm{s}$, the displacement time history and phase diagram of the VIV response of the riser system show the standard periodic vibration law (see Figure 7). The FFT spectrum contains only the frequency component close to the $3^{\text {rd }}$-order structural hydroelastic modal frequency. The maximum displacement envelope diagram also shows the $3^{\text {rd }}$ order resonance mode shape, which implies that the $3^{\text {rd }}$-order vortex-induced resonance occurs in the riser system.

4.1.2. Almost Periodic Vibration. From the $g$ bifurcation diagram shown in Figure 2, it can be found that the flow velocity ranges in which almost periodic vibration occurs are very wide. The almost periodic vibration response of the riser system also varies in different regions. Three typical flow 


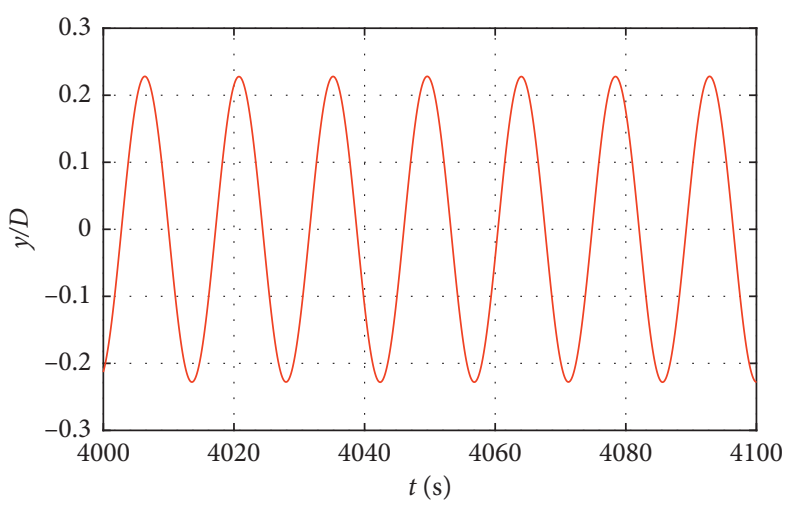

(a)

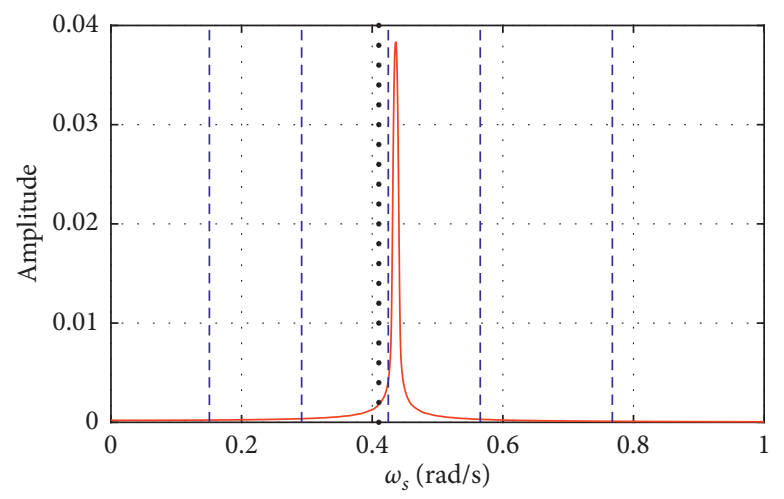

(c)

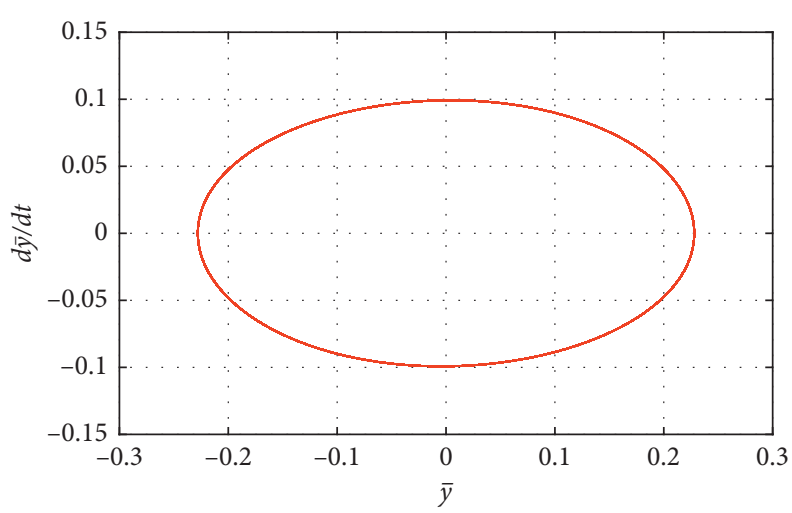

(b)

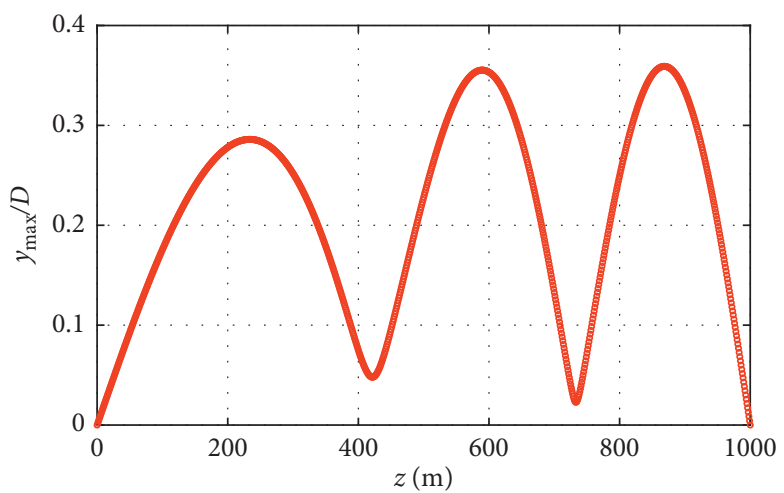

(d)

Figure 7: The VIV response of the riser system at surface flow velocity $U=0.15 \mathrm{~m} / \mathrm{s}$. (a) Displacement time history. (b) Phase diagram of displacement and velocity. (c) FFT spectrum. (d) Maximum displacement envelope.

velocities are selected to calculate the VIV response of the riser system as follows:

(1) $U=0.08 \mathrm{~m} / \mathrm{s}$ and $\Omega_{f e}=0.218 \mathrm{rad} / \mathrm{s}$

When $U=0.08 \mathrm{~m} / \mathrm{s}$, combined with the displacement time history response shown in Figure 8(a) and the Poincaré section in Figure 8(b), the VIV response of the riser system can be identified as the almost periodic motion. It can be seen from the FFT spectrum that $\Omega_{f e}$ appears between the first and second structural hydroelastic modal frequencies. Moreover, the frequency components excited in Figure $8(\mathrm{c})$ are the same as the first two order frequencies of the structural hydroelastic mode, and the line spectrum of the first order takes dominance. The maximum displacement shown in Figure 8(d) envelopes the transitional modes of the first two orders.

(2) $U=0.13 \mathrm{~m} / \mathrm{s}$ and $\Omega_{f e}=0.355 \mathrm{rad} / \mathrm{s}$

When $U=0.13 \mathrm{~m} / \mathrm{s}$, the displacement time history and phase diagram of the VIV response of the riser system are more complicated. The VIV response can still be categorized as almost periodic motion by looking at the Poincare section in Figure 9(b). There are four main frequency components in the FFT spectrum of Figure 9(c), which correspond to the first four orders of the structural hydroelastic modal frequencies. It can be shown that vortex shedding excites the first four modes of the riser system simultaneously, and the second and third components are more significant. Since $\Omega_{f e}$ is between the secondorder and third-order frequencies of the structural hydroelastic mode, the maximum displacement shown in Figure 9(d) envelopes the transitional mode shape of the $2^{\text {nd }}$ and $3^{\text {rd }}$ order.

4.1.3. Bistable Phenomenon of the Riser System. The transitional region between periodic to almost periodic motions of the riser system shown in Figure 2 is characterized by sudden jump and hysteresis of system response. Such dynamics corresponds to bi-stable phenomena of nonlinear systems. The bistable state includes the coexistence of different almost periodic motions. Also, it may have periodic motion and the coexistence of almost periodic motions.

In the range from $0.2 \mathrm{~m} / \mathrm{s}$ to $0.224 \mathrm{~m} / \mathrm{s}$, from the point discontinuity, it can be judged that subcritical Hopf bifurcation occurs in the riser system. Three typical flow velocities are selected from the bistable regions to study the VIV response of the riser system as follows:

(1) $U=0.138 \mathrm{~m} / \mathrm{s}$ and $\Omega_{f e}=0.377 \mathrm{rad} / \mathrm{s}$

When $U=0.138 \mathrm{~m} / \mathrm{s}$, two stable almost periodic motions coexist in the VIV response of riser system, 


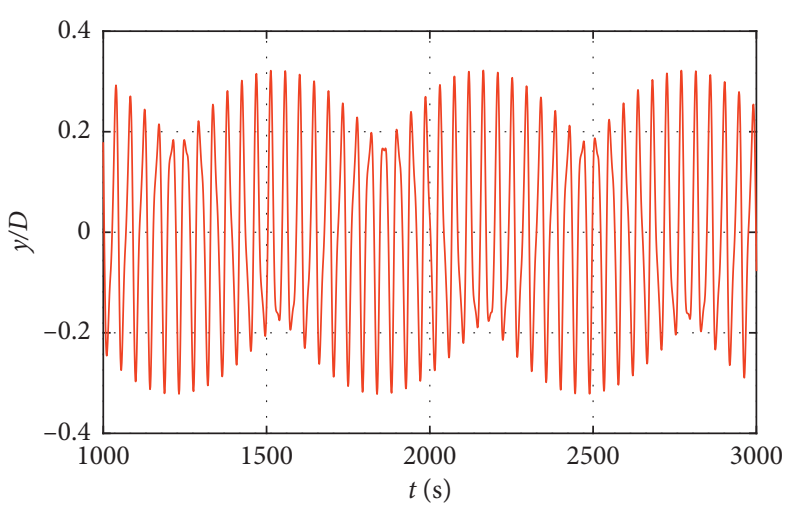

(a)

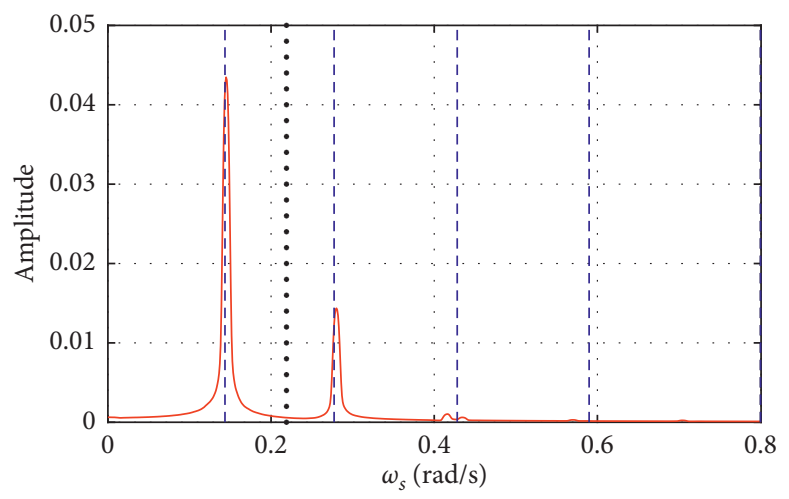

(c)

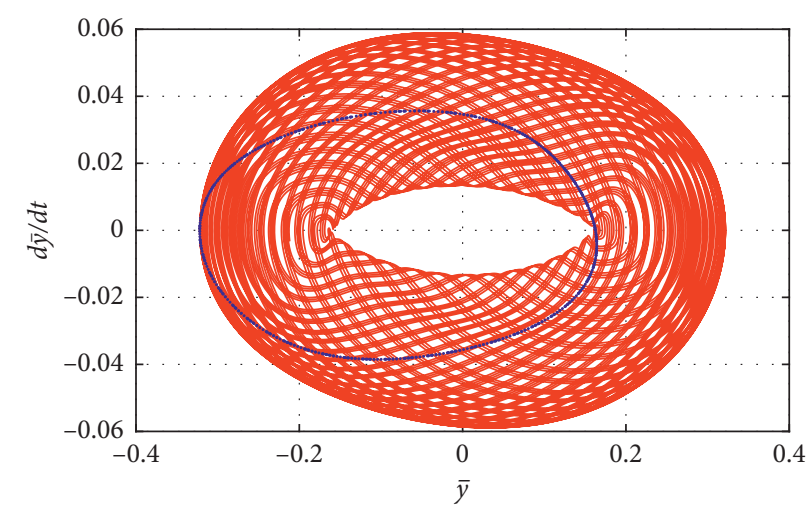

(b)

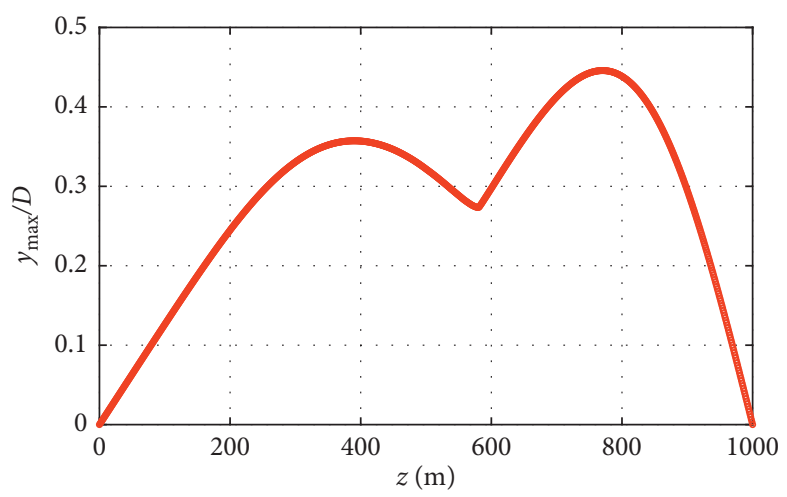

(d)

FIgure 8: The VIV response of the riser system at surface flow velocity $U=0.08 \mathrm{~m} / \mathrm{s}$. (a) Displacement time history. (b) Phase diagram of displacement and velocity. (c) FFT spectrum. (d) Maximum displacement envelope.

and their maximum displacement envelope are similar as the $3^{\text {rd }}$-order mode shape. Figure $10(\mathrm{c})$ shows that the dominated frequency components of these two motions are almost overlapped and close to the $3^{\text {rd }}$-order structural hydroelastic modal frequency. One subtle difference can be observed that the red line also includes obvious frequency components corresponding to both $1^{\text {st }}$ - and $2^{\text {nd }}$-order structural hydroelastic modes.

(2) $U=0.162 \mathrm{~m} / \mathrm{s}$ and $\Omega_{f e}=0.443 \mathrm{rad} / \mathrm{s}$

When $U=0.162 \mathrm{~m} / \mathrm{s}, \Omega_{f e}$ is slightly larger than the third-order structural hydroelastic modal frequency, and the VIV response occurs bistable phenomenon. For the red line case, it can be seen that the vibration response of the riser system is still periodic. The FFT spectrum contains only one frequency component close to the $3^{\text {rd }}$-order structural hydroelastic modal frequency. The maximum displacement envelope is identical with the $3^{\text {rd }}$-order resonance mode shape.

The motion pattern corresponding to the black curve in Figure 11(a) is more complex compared with the previous discussions. Its phase diagram is torus. By further viewing a representative motion (see Figure 11(b)), the VIV response can be identified as almost periodic. The first five orders of the structural hydroelastic modal frequency of the riser system appear in the FFT spectrum, and the maximum displacement envelope of the riser shows transitional mode shape from the $3^{\text {rd }}$-order to $4^{\text {th }}$-order vibration.

(3) $U=0.21 \mathrm{~m} / \mathrm{s}$ and $\Omega_{f e}=0.574 \mathrm{rad} / \mathrm{s}$

When $U=0.21 \mathrm{~m} / \mathrm{s}, \Omega_{f e}$ is close to the fourth-order structural hydroelastic modal frequency. Under this flow velocity, the system enters the bistable region from $4^{\text {th }}$-order resonance to $5^{\text {th }}$-order vibration. The VIV response of the riser system in this region occur bistable phenomenon with large jump. The riser system still shows the $4^{\text {th }}$-order mode as the red curve in Figure 12(a) shows. Such highly periodic motion is reflected as the blue dot in the red elliptical Poincare section in Figure 12(b). The frequency component excited in the FFT spectrum is close to the $4^{\text {th }}$-order structural hydroelastic modal frequency, and the maximum displacement envelope shows the $4^{\text {th }}$-order primary resonance mode shape.

For the decomposed motion plotted as black curve in Figure 12(a), the corresponding motion in the Poincaré section is a green closed curve in the black elliptical ring. This implies that the VIV response is almost periodic. The frequency components excited in the FFT spectrum are similar 


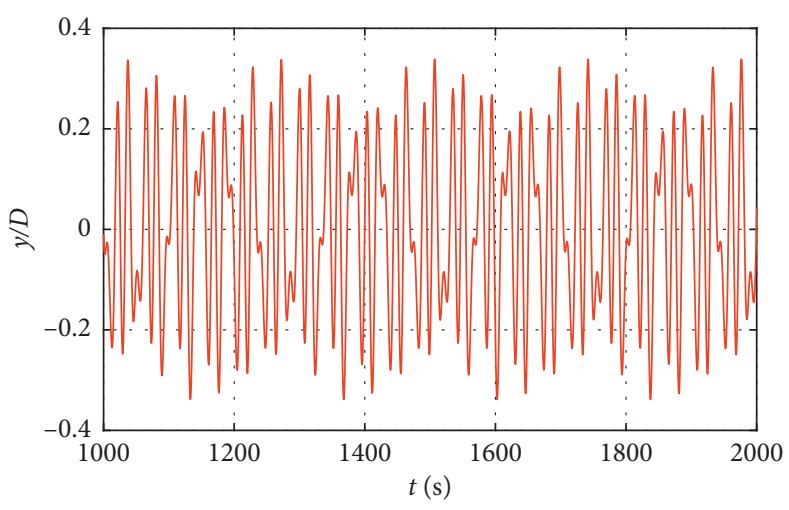

(a)

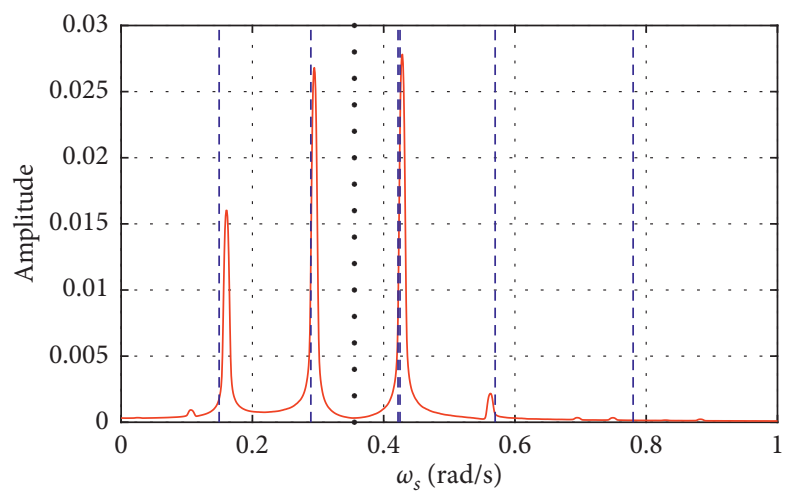

(c)

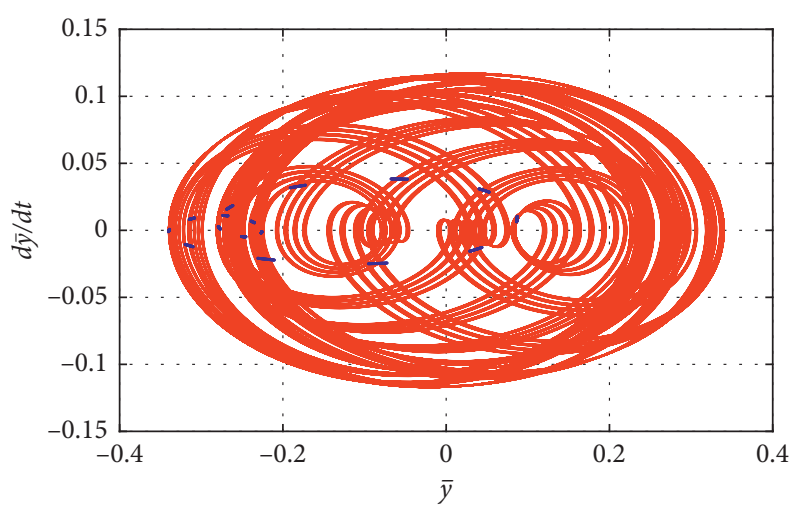

(b)

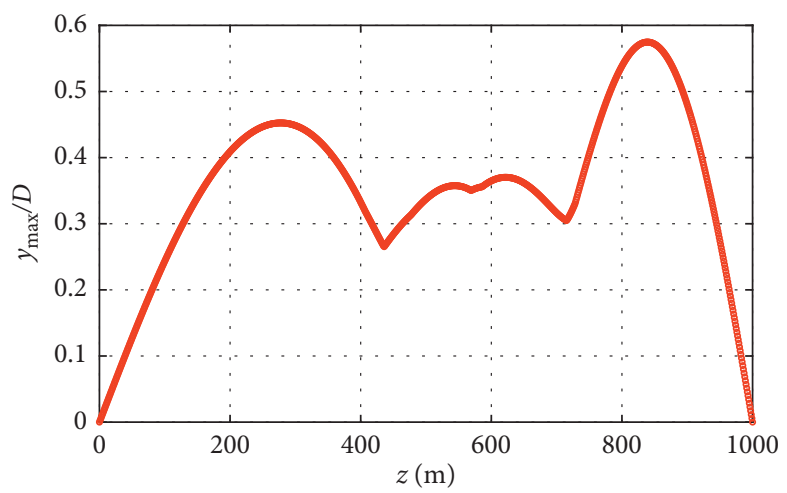

(d)

FIgURE 9: The VIV response of the riser system at surface flow velocity $U=0.13 \mathrm{~m} / \mathrm{s}$. (a) Displacement time history. (b) Phase diagram of displacement and velocity. (c) FFT spectrum. (d) Maximum displacement envelope.

to the $1^{\text {st }}$-order and $5^{\text {th }}$-order structural hydroelastic modal frequencies, and the maximum displacement envelope shows the $5^{\text {th }}$-order vibration mode shape.

4.2. Comparison between the $3 D$ Spectrum and Eigenvalue Changes. In order to better show the variation trend of the FFT spectrum of the fluid-structure coupled riser system, normalized three-dimensional spectrum contours with increasing and decreasing flow velocity are discussed in this section. The 3D spectrum contours are then being mapped to the imaginary part of the eigenvalue of the riser system, as shown in Figures 13(a) and 13(b). In Figure 13 the purple red line is the imaginary part of the eigenvalue. The contour plot shows the frequency component of the riser system excited at each flow velocity. The black line is the equivalent vortex shedding frequency $\Omega_{f e}$ at the riser midpoint.

It can be seen from Figure 13 that with increasing flow velocity, modal frequencies are sequentially excited for each order, and the amplitudes of each mode are positively correlated with the structural hydroelastic modal frequencies. Combined with the response phenomenon of the riser system discussed in previous subsections, it can be found that when $\Omega_{f e}$ reaches to a structural hydroelastic resonance frequency, the corresponding mode will dominate the system VIV response. This further suggests that the equivalent velocity $U e$ and the equivalent vortex shedding frequency $\Omega_{f e}$ are the key parameters when analyzing the VIV response of riser in shear flow. In addition, for the response frequency components of the riser structure and the wake in the primary resonance region of each order, there are obvious shifts in the position between Figures 13(a) and 13(b) at the same flow velocity, which also confirm that the VIV of the riser system will appear bistable phenomenon.

It can be seen from Figure 13 that when the flow velocity is small, the wake action dominates the coupled motion of the riser system. The main frequency component of the VIV is consistent with the frequency of each order wake-induced mode. With increasing flow velocity, structural motion begins to dominate the overall VIV response, and the primary resonant modes of the riser structure are excited in turn. When the flow velocity continues to increase, the wake frequency components become obvious again.

After analyzing Figures 13(a) and 13(b), we can find that under one flow velocity, the flow force may excite different structural response frequencies of the riser system. This is especially pronounced that the bistable phenomenon occurs easily at the critical point between adjacent resonant frequencies. Such finding accords with the condition in 


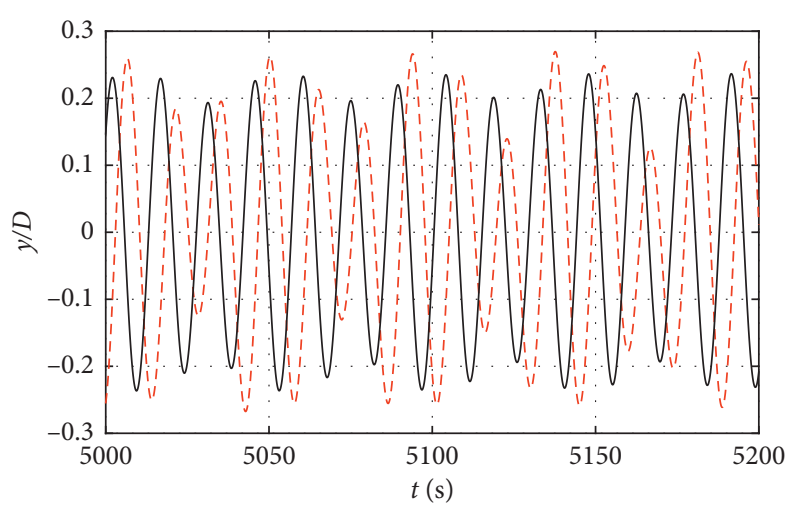

(a)

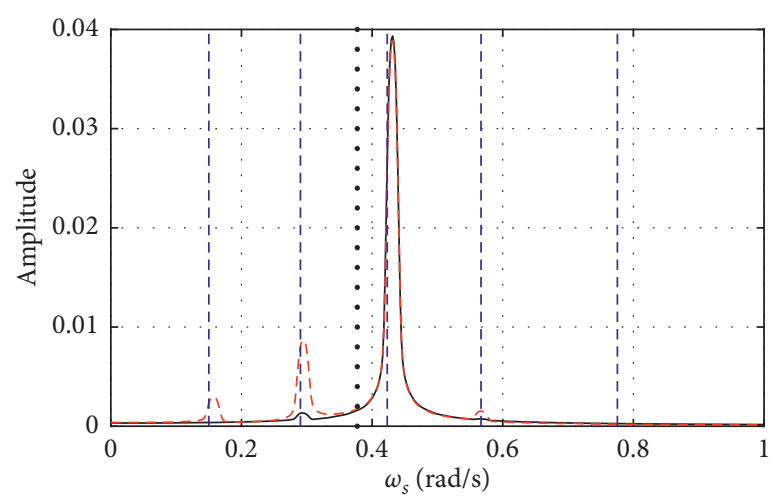

(c)

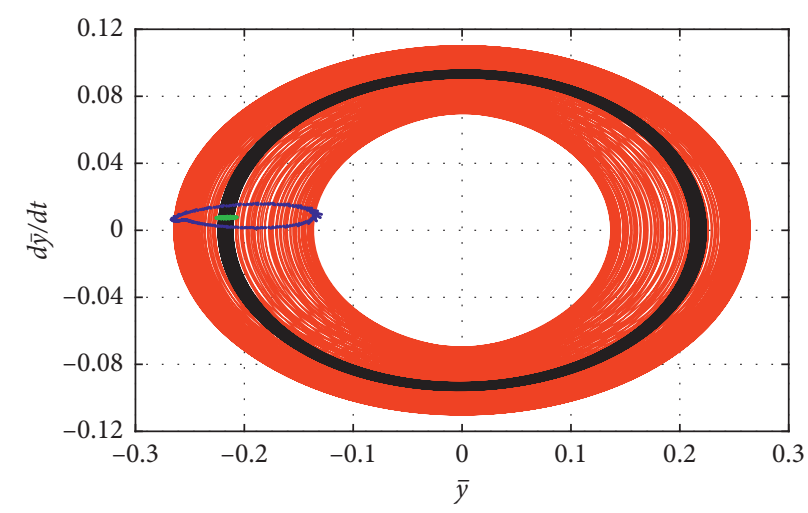

(b)

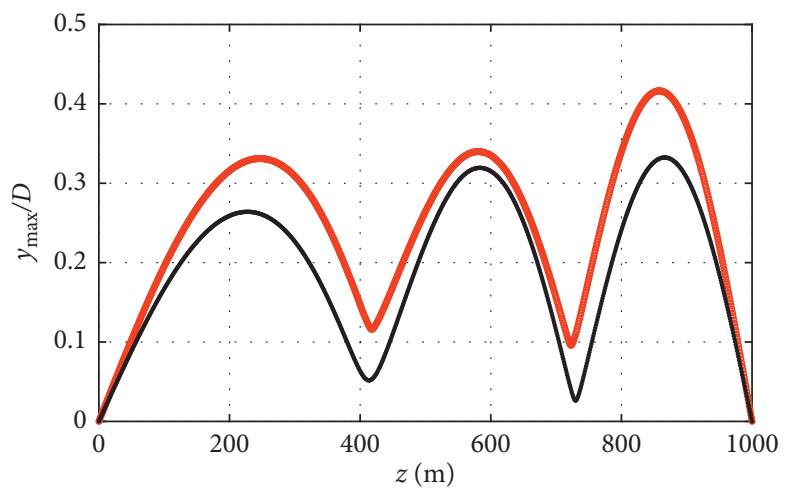

(d)

FIgURE 10: The VIV response of the riser system at surface flow velocity $U=0.138 \mathrm{~m} / \mathrm{s}$. (a) Displacement time history. (b) Phase diagram of displacement and velocity. (c) FFT spectrum. (d) Maximum displacement envelope.

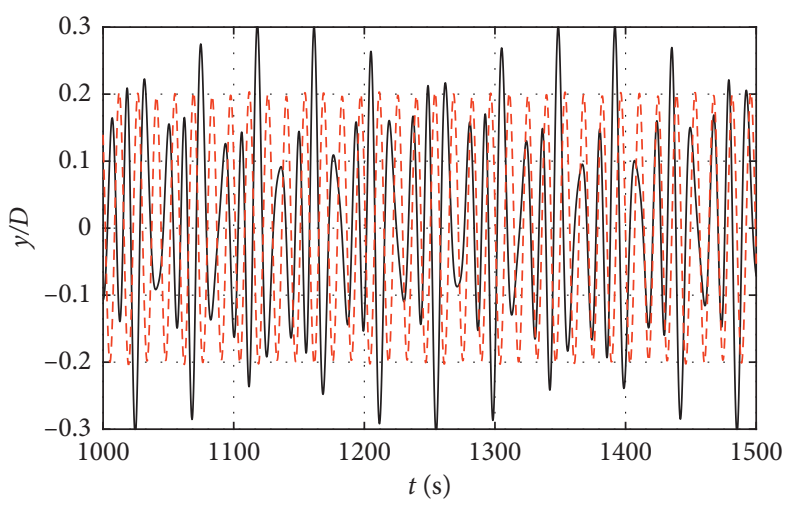

(a)

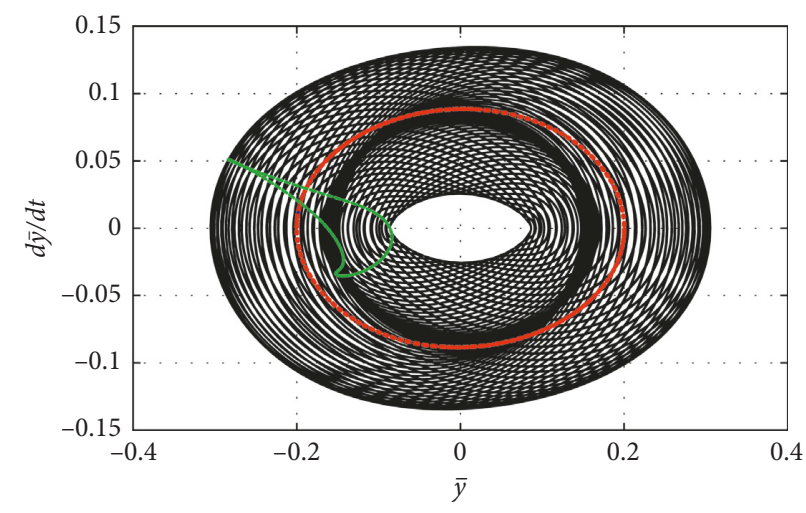

(b)

FIgURE 11: Continued. 


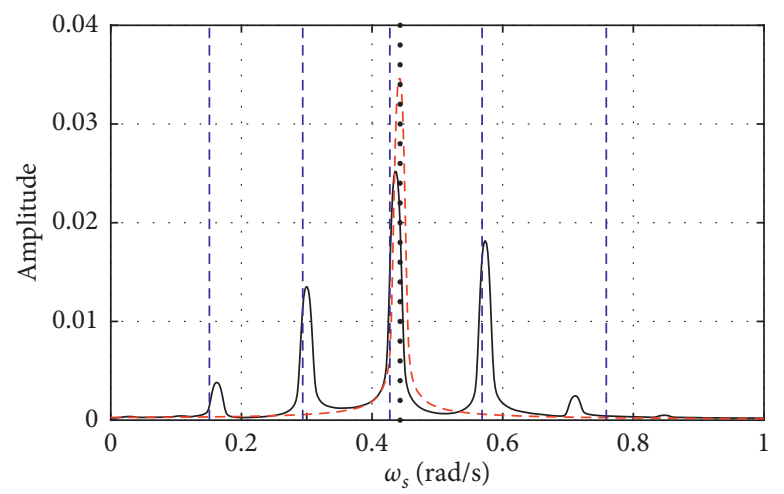

(c)

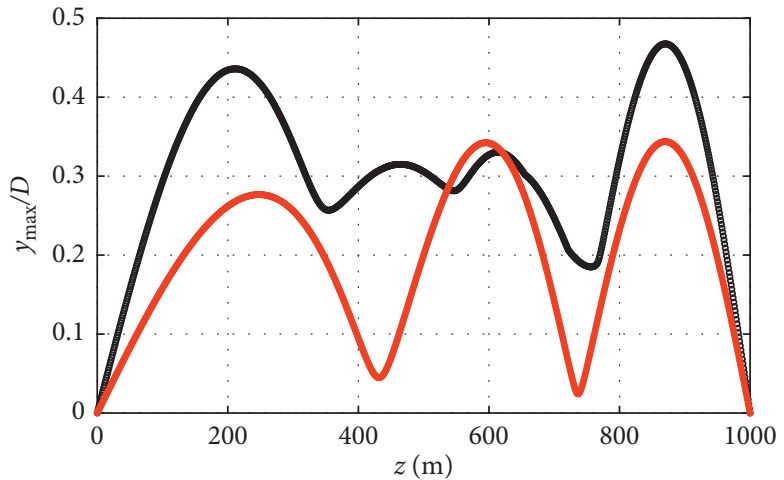

(d)

FIgURE 11: The VIV response of the riser system at surface flow velocity $U=0.162 \mathrm{~m} / \mathrm{s}$. (a)Displacement time history. (b) Phase diagram of displacement and velocity. (c) FFT spectrum. (d) Maximum displacement envelope.

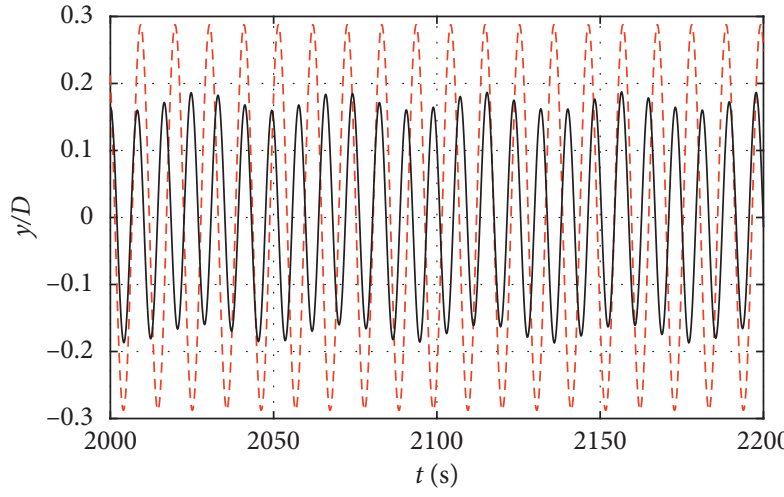

(a)

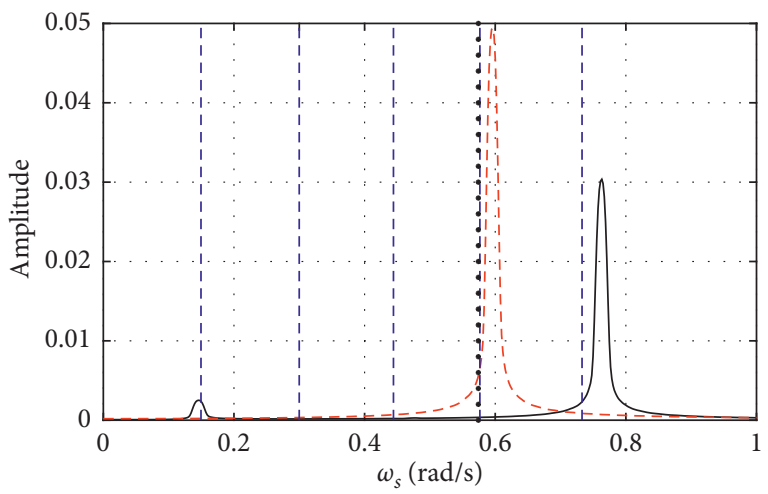

(c)

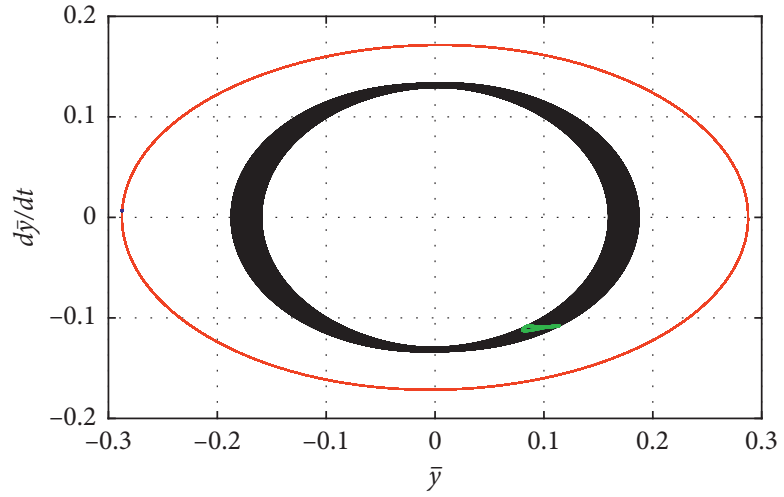

(b)

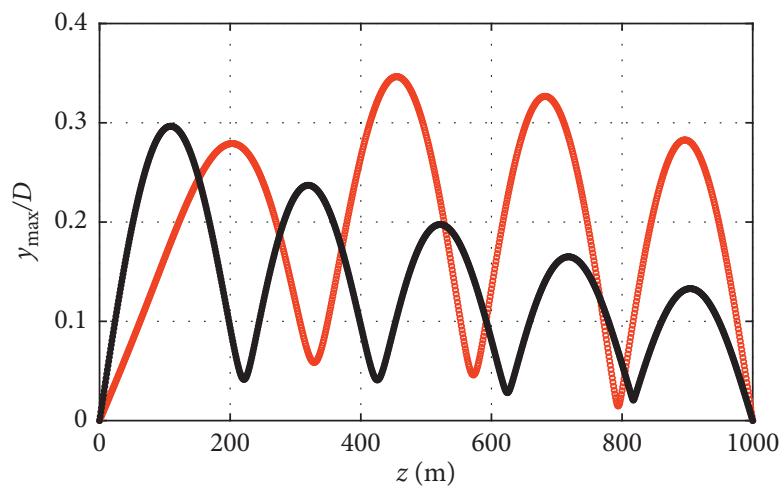

(d)

Figure 12: The VIV response of the riser system at surface flow velocity $U=0.21 \mathrm{~m} / \mathrm{s}$. (a)Displacement time history. (b) Phase diagram of displacement and velocity. (c) FFT spectrum. (d) Maximum displacement envelope.

Figure 2 that the bistable phenomena occur at both ends of periodic solution. For nonlinear slender riser structure, the $1^{\text {st }}$-order structural hydroelastic modal frequency of the riser system gradually decreases to zero as the flow velocity keeps increasing in Figure 13. This is due to the buckling of strut caused by the axial force. It is worthy to mention that as the calculated results are based on the $5^{\text {th }}$-order Galerkin discretization in this paper, the $5^{\text {th }}$-order VIV response of the riser system is less accurate due to discretization error in the numerical scheme. 


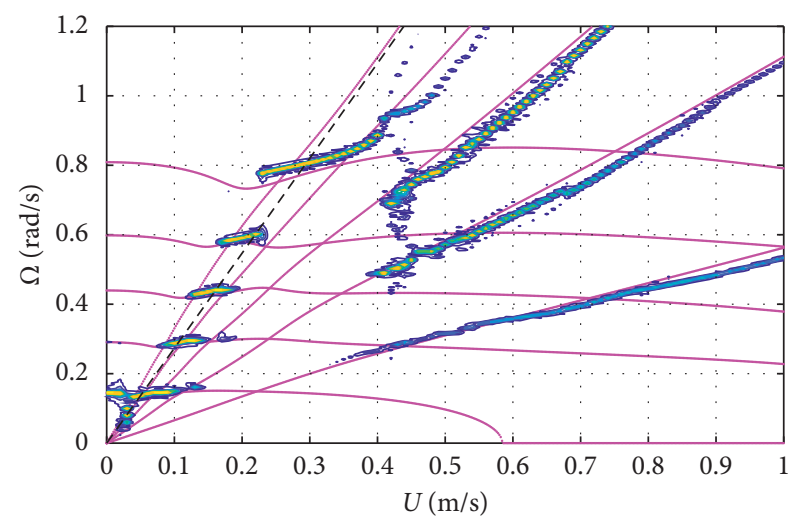

(a)

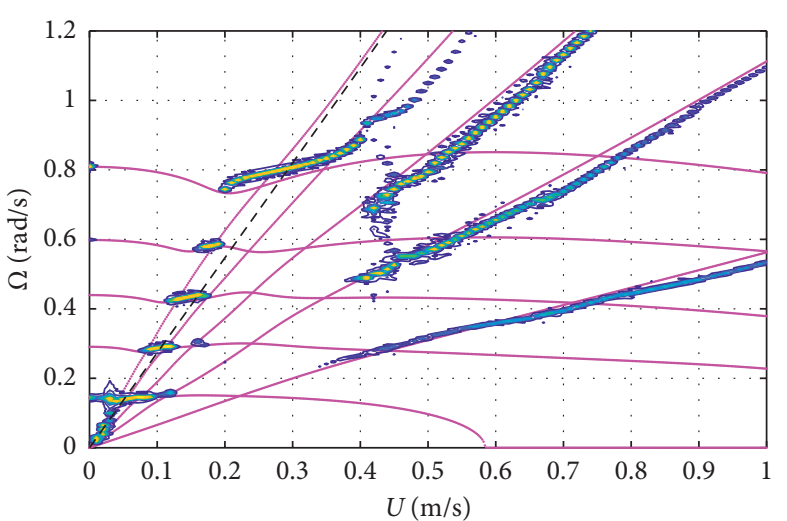

(b)

FIgURE 13: Comparison between the 3D spectrum contour and the imaginary part of the eigenvalues. (a) Calculation result with the condition of flow velocity increasing. (b) Calculation result with the condition of flow velocity decreasing.

\section{Conclusion}

Based on the Van der Pol wake oscillator model, the nonlinear dynamic characteristics of the vortex-induced vibration of the top tension riser in linear shear flow are studied. The conclusions are as follows:

(1) The bifurcation diagram of the local maximum value of the VIV cross-flow dimensionless displacement of the riser with respect to the flow velocities is obtained. Compared with the amplitude response diagram, the bifurcation diagram can more clearly judge the nonlinear dynamic behavior of the system. It can be seen from the bifurcation diagram that with the flow velocity increasing, the almost periodical vibration and periodical vibration of the riser system will appear alternately. The bistable behavior and Hopf bifurcation phenomenon such as hysteresis and jump will occur in the transition region between periodic and almost periodic vibrations. In the bistable region, the steady-state response of the riser VIV will be different under different initial conditions.

(2) It is found out that when flow velocity is less than $0.2 \mathrm{~m} / \mathrm{s}$, the riser system response will form the torus-like almost periodic motion with multimode interaction between adjacent periodic motions along the bifurcation path. The torus Hopf bifurcation may appear during the transition process between periodic and almost periodic motions. When the flow velocity is within the range of $0.2 \mathrm{~m} / \mathrm{s}$ to $0.22 \mathrm{~m} / \mathrm{s}$, the vibration response of the riser system have two large jumps with sudden increasing or decreasing of amplitude. The vibration response of the riser system in this region is subcritical Hopf bifurcation. There are bistable behaviors in all these regions.

(3) Due to the influence of nonlinear factors and damping, the structural hydroelastic modal frequency of the riser fluid-structure coupled system may vary with respect to flow velocity. Combined with the analysis of the $3 \mathrm{D}$ frequency spectrogram, it can be deduced that with increasing flow velocity, the riser system will have vortex-induced resonance frequency lock-in bands near the natural frequencies of each mode. Overlapping between adjacent modes may occur for the critical velocity range, which results in bistable regions. In some bistable regions, VIV responses would be quite different. This offers useful insight for fatigue analysis of risers. For practical engineering problems, improved simulations that enable the capture of richer nonlinear dynamics can be carried out with proper choice of Galerkin discretization order.

\section{Data Availability}

The data used to support the findings of this study are available from the corresponding author upon request.

\section{Conflicts of Interest}

The authors declare that they have no conflicts of interest.

\section{Acknowledgments}

The study was supported by the National Program on Key Basic Research Project (no. 2014CB046805) and the National Natural Science Foundation of China (no. 11672349).

\section{References}

[1] J. Cai, Y.-X. You, W. Li, Z. M. Shi, and Y. Qu, “The VIV characteristics of deep-sea risers with high aspect ratio in a uniform current profile," Journal of Hydrodynamics, vol. 25, no. 1, pp. 50-58, 2010.

[2] W.-M. Chen, Y.-Q. Fu, and S.-X. Guo, "Review on fluid-solid coupling and dynamic response of vortex-induced vibration of slender ocean cylinders," Advances in Mechanics, vol. 47, no. 1, pp. 25-91, 2017.

[3] E. Huse, G. Kleiven, and F. G. Nielsen, "Large scale model testing of deep sea risers," in Proceedings of the Offshore Technology Conference, Houston, Texas, USA, May 1998. 
[4] E. Huse, G. Kleiven, and F. G. Nielsen, "VIV-induced axial vibrations in deep sea risers," in Proceedings of the Offshore Technology Conference, Houston, Texas, USA, May 1999.

[5] S. Dong and G. E. Karniadakis, "DNS of flow past a stationary and oscillating cylinder at," Journal of Fluids and Structures, vol. 20, no. 4, pp. 519-531, 2005.

[6] H. Al-Jamal and C. Dalton, "Vortex induced vibrations using large eddy simulation at a moderate reynolds number," Journal of Fluids and Structures, vol. 19, no. 1, pp. 73-92, 2004.

[7] C. Yamamoto, J. R. Meneghini, F. Saltara, R. A. Fregonesi, and J. A. Ferrari, "Numerical simulations of vortex-induced vibration on flexible cylinders," Journal of Fluids and Structures, vol. 19, no. 4, pp. 467-489, 2004.

[8] W.-M. Chen, Z.-Q. Zheng, L.-W. Zhang et al., "Vortex-induced vibration of deepwater flexible riser experencing internal-wave-induced shear flow," Engineering Mechanics, vol. 28 , no. 12 , pp. 250-256, 2011.

[9] F. J. Huera Huartea, P. W. Bearman, and J. R. Chaplin, "On the force distribution along the axis of a flexible circular cylinder undergoing multi-mode vortex-induced vibrations," Journal of Fluids and Structures, vol. 22, no. 6-7, pp. 897-903, 2006.

[10] H. Lie and K. E. Kaasen, "Modal analysis of measurements from a large-scale VIV model test of a riser in linearly sheared flow," Journal of Fluids and Structures, vol. 22, no. 4, pp. 557-575, 2006.

[11] D. Lucor, H. Mukundan, and M. S. Triantafyllou, "Riser modal identification in CFD and full-scale experiments," Journal of Fluids and Structures, vol. 22, no. 6-7, pp. 905-917, 2006.

[12] Y. Tang, T. Yang, and B. Fang, "Fractional dynamics of fluidconveying pipes made of polymer-like materials," Acta Mechanica Solida Sinica, vol. 31, no. 2, pp. 243-258, 2018.

[13] M. H. Ghayesh, M. P. Païdoussis, and M. Amabili, "Nonlinear dynamics of cantilevered extensible pipes conveying fluid," Journal of Sound and Vibration, vol. 332, no. 24, pp. 64056418, 2013.

[14] Y. Modarres-Sadeghi and M. P. Païdoussis, "Chaotic oscillations of long pipes conveying fluid in the presence of a large end-mass," Computers \& Structures, vol. 122, pp. 192-201, 2013.

[15] W. Yang, Z. Ai, X. Zhang, X. Chang, and R. Gou, "Nonlinear dynamics of three-dimensional vortex-induced vibration prediction model for a flexible fluid-conveying pipe," International Journal of Mechanical Sciences, vol. 138-139, pp. 99-109, 2018.

[16] J. Duan, K. Chen, Y. You, and J. Li, "Numerical investigation of vortex-induced vibration of a riser with internal flow," Applied Ocean Research, vol. 72, pp. 110-121, 2018.

[17] L. Wang, T. L. Jiang, H. L. Dai, and Q. Ni, "Three-dimensional vortex-induced vibrations of supported pipes conveying fluid based on wake oscillator models," Journal of Sound and Vibration, vol. 422, pp. 590-612, 2018.

[18] S. Meng, X. Zhang, C. Che, and W. Zhang, "Cross-flow vortex-induced vibration of a flexible riser transporting an internal flow from subcritical to supercritical," Ocean Engineering, vol. 139, pp. 74-84, 2017.

[19] R. Hartlen and I. G. Currie, "Lift-oscillator model of vortexinduced vibration," urnal of the Engineering Mechanics Division, vol. 96, no. 5, pp. 577-591, 1970.

[20] L. Mathelin and E. de Langre, "Vortex-induced vibrations and waves under shear flow with a wake oscillator model," $E$ uropean Journal of Mechanics-B/Fluids, vol. 24, no. 4, pp. 478-490, 2005.
[21] M. L. Facchinetti, E. de Langre, and F. Biolley, "Vortex shedding modeling using diffusive van der pol oscillators," Comptes Rendus Mécanique, vol. 330, no. 7, pp. 451-456, 2002.

[22] M. L. Facchinetti, E. de Langre, and F. Biolley, "Coupling of structure and wake oscillators in vortex-induced vibrations," Journal of Fluids and Structures, vol. 19, no. 2, pp. 123-140, 2004.

[23] M. L. Facchinetti, E. de Langre, and F. Biolley, "Vortex-induced travelling waves along a cable," European Journal of Mechanics-B/Fluids, vol. 23, no. 1, pp. 199-208, 2004.

[24] H.-Y. Guo and M. Lou, "Effect of internal flow on vortexinduced vibration of risers," Journal of Fluids and Structures, vol. 24, no. 4, pp. 496-504, 2008.

[25] Y.-G. Tang, W.-D. Shao, J. Zhang, L. Y. Wang, and L. Gui, "Dynamic response analysis for coupled parametric vibration and vortex-induced vibration of top-tensioned riser in deepsea," Engineering Mechanics, vol. 30, no. 5, pp. 282-286, 2013.

[26] J. B. V. Wanderley, S. H. Sphaier, and C. Levi, "A two-dimensional numerical investigation of the hysteresis effect on vortex induced vibration on an elastically mounted rigid cylinder," Journal of Offshore Mechanics and Arctic Engineering, vol. 134, no. 2, p. 21801, 2012.

[27] N. V. Yogeswaran, V. Yogeswaran, S. Sen, and S. Mittal, "Free vibrations of an elliptic cylinder at low reynolds numbers," Journal of Fluids and Structures, vol. 51, pp. 55-67, 2014.

[28] H. L. Dai, L. Wang, Q. Qian, and Q. Ni, "Vortex-induced vibrations of pipes conveying pulsating fluid," Ocean Engineering, vol. 77, pp. 12-22, 2014.

[29] Y.-C. Wang, Z.-Q. Wu, and X.-Y. Zhang, "Vortex-induced vibration response bifurcation analysis of top-tensioned riser based on the model of variable lift coefficient," Mathematical Problems in Engineering, vol. 2018, Article ID 6491517, 11 pages, 2018.

[30] I. K. Chatjigeorgiou, "On the parametric excitation of vertical elastic slender structures and the effect of damping in marine applications," Applied Ocean Research, vol. 26, no. 1-2, pp. 23-33, 2004.

[31] H. L. Dai, L. Wang, Q. Qian, and Q. Ni, "Vortex-induced vibrations of pipes conveying fluid in the subcritical and supercritical regimes," Journal of Fluids and Structures, vol. 39, pp. 322-334, 2013.

[32] R. Violette, E. de Langre, and J. Szydlowski, "Computation of vortex-induced vibrations of long structures using a wake oscillator model: comparison with DNS and experiments," Computers \& Structures, vol. 85, no. 11-14, pp. 1134-1141, 2007.

[33] R. Shabani, S. Tariverdilo, and H. Salarieh, "Nonlinear vibrations and chaos in floating roofs," Journal of Computational and Nonlinear Dynamics, vol. 7, no. 2, Article ID 0210122, 2012.

[34] M. Zamanian and S. E. Khadem, "Stability analysis of an electrically actuated microbeam using the Melnikov theorem and Poincaré mapping," Proceedings of the Institution of Mechanical Engineers, Part C: Journal of Mechanical Engineering Science, vol. 225, no. 2, pp. 488-497, 2011.

[35] K. Tsumoto, H. Kitajima, T. Yoshinaga, K. Aihara, and H. Kawakami, "Bifurcations in morris-lecar neuron model," Neurocomputing, vol. 69, no. 4-6, pp. 293-316, 2006. 


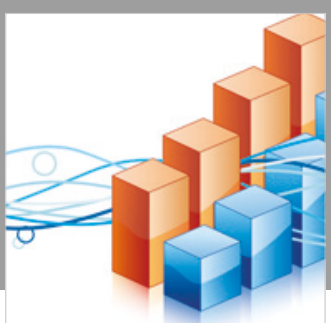

Advances in

Operations Research

\section{-n-m}
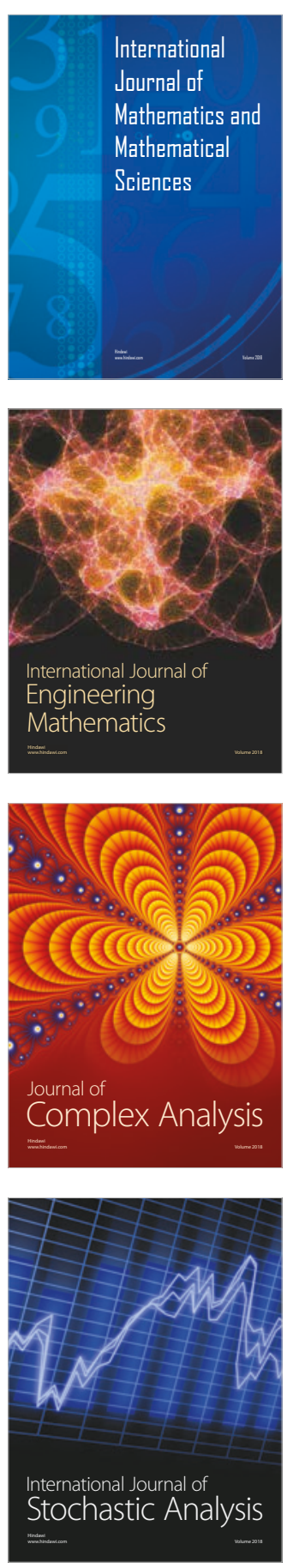
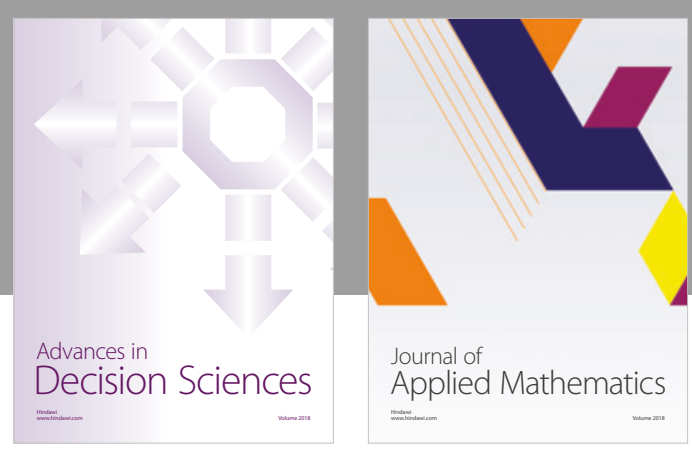

Journal of

Applied Mathematics
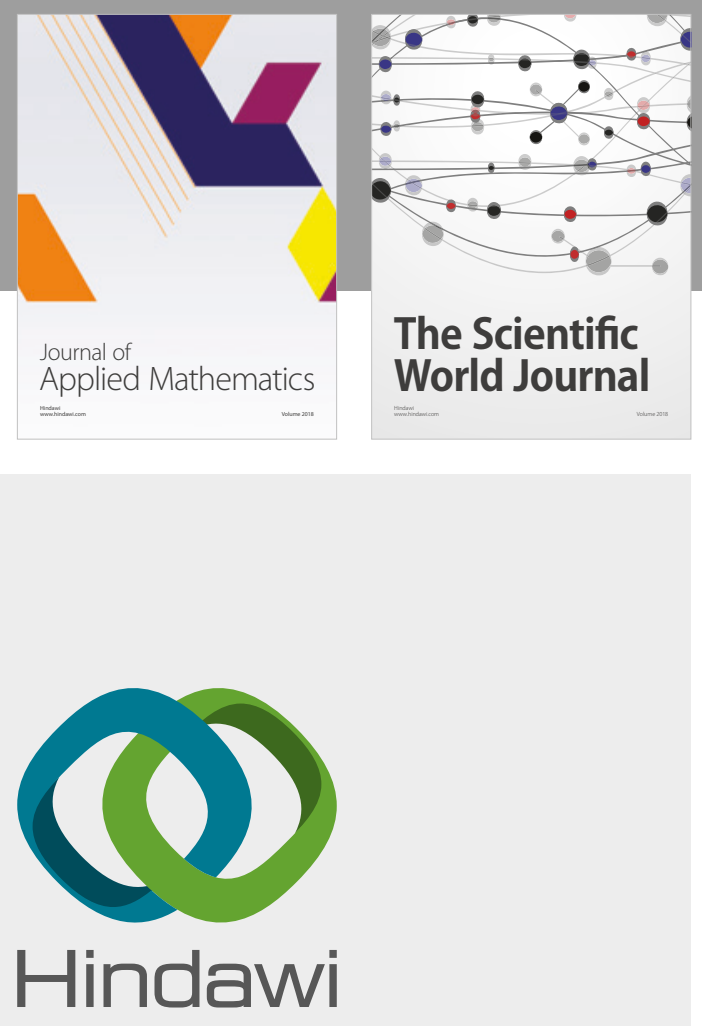

Submit your manuscripts at

www.hindawi.com

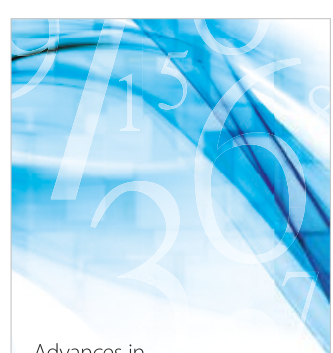

Advances in
Numerical Analysis
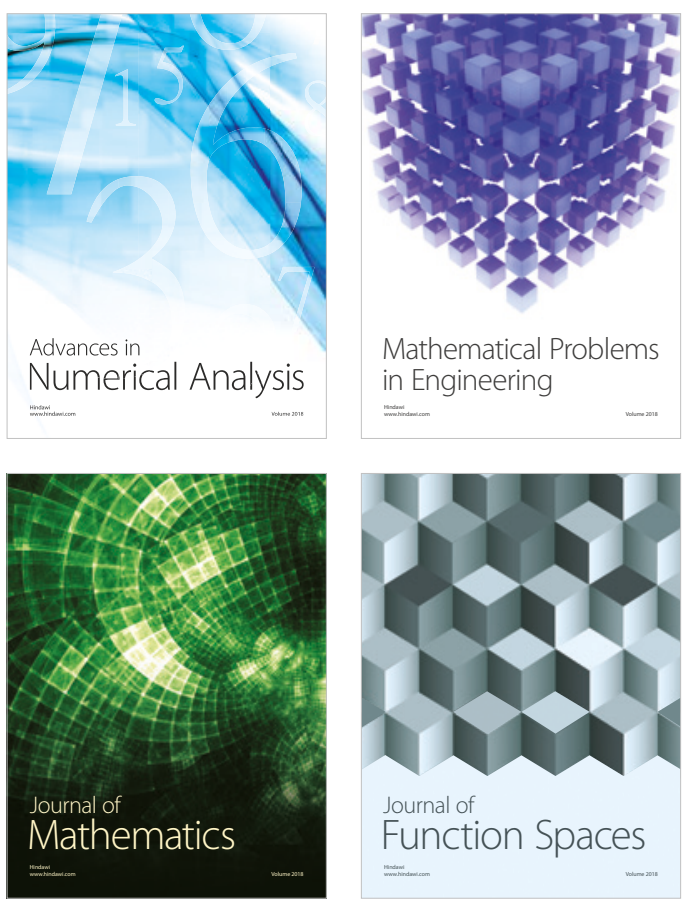

Mathematical Problems in Engineering

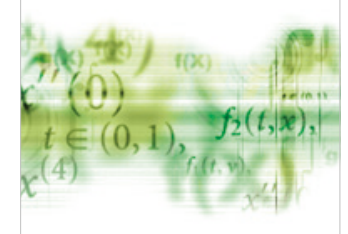

International Journal of

Differential Equations

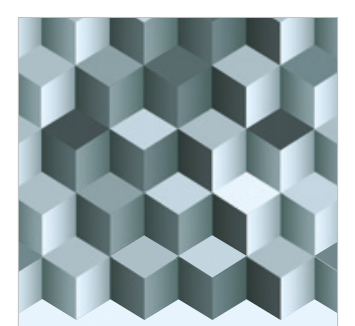

Journal of

Function Spaces

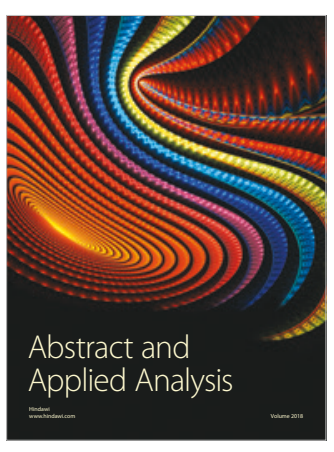

The Scientific

World Journal

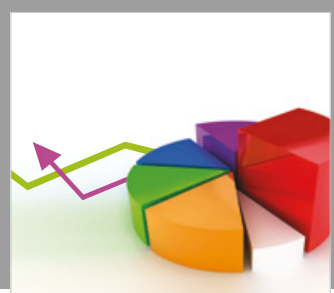

Journal of

Probability and Statistics
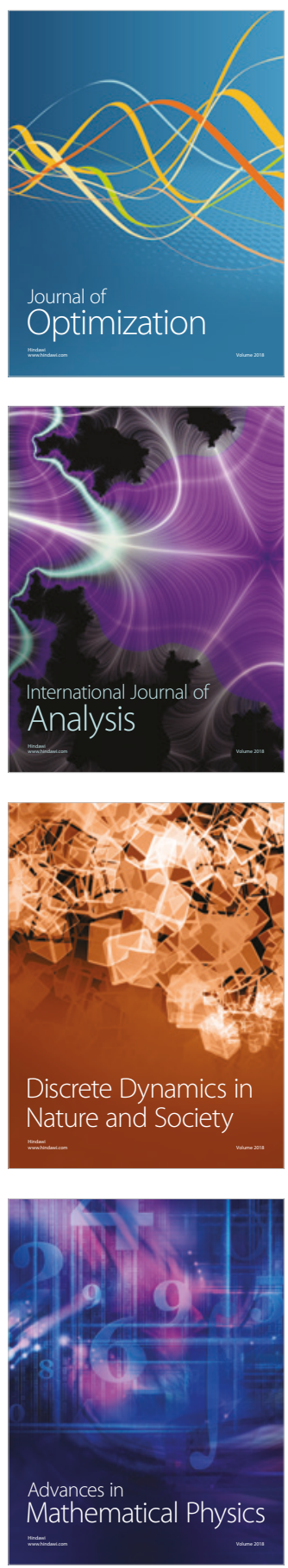